\title{
Challenging collaboration of general practitioners and specialists for patients with complex chronic conditions: A scoping review to identify improvement strategies
}

\section{Rebecca Tomaschek ( $\nabla$ rebecca.tomaschek@iham-cc.ch )}

Research Institute for Primary Care and Health Sciences: Keele University School of Primary Community and Social Care https://orcid.org/0000-0003-4626-248X

\section{Patricia Lampart}

Swiss Paraplegic Centre: Schweizer Paraplegiker Zentrum

\section{Anke Scheel-Sailer}

Swiss Paraplegic Centre: Schweizer Paraplegiker Zentrum

\section{Armin Gemperli}

Swiss Paraplegic Research

\section{Christoph Merlo}

Institute of Primary and Community Care Lucerne

\section{Stefan Essig}

Institute of Primary and Community Care Lucerne

\section{Research article}

Keywords: complex chronic disease, long-term care, care provision, primary care, specialized care, collaboration

Posted Date: February 9th, 2021

DOl: https://doi.org/10.21203/rs.3.rs-191654/v1

License: (a) This work is licensed under a Creative Commons Attribution 4.0 International License. Read Full License 


\section{Abstract}

\section{Background}

Collaboration between medical specialists and general practitioners (GPs) is well known from daily practice, but research on the topic is rather scarce. This scoping review summarizes role distributions and components of this collaboration that have potential for improvement for the care of patients with complex chronic conditions.

\section{Methods}

Scoping review as a knowledge synthesis for components of collaboration and role distributions between medical specialists and GPs in intervention studies. The PubMed database was searched for literature from 2010-2020.

\section{Results}

Literature search and reference screening generated 2,174 articles. Of these, 30 articles originating from 22 unique projects were included in our synthesis. These projects focus on different patient populations and take diverse approaches to improve collaboration within an intervention. The GP is most commonly in charge of patient management and extends the scope of practice. The specialist provides support when needed. Clear definition of roles and responsibilities, resources for knowledge exchange and raising awareness through education from specialists for specific health conditions are commonly utilized interventions. Typically, combinations of process and system changes that address communication and coordination issues are applied. Most interventions improve provider and patient satisfaction, health outcomes, and reduce fragmentation of patient care.

\section{Conclusion}

Interventions to improve collaboration between GPs and medical specialists seem promising. Further efforts should be made to test and apply the findings in broad clinical practice.

\section{Background}

Collaboration between specialists and general practitioners (GPs) is well known from daily practice, but research on the topic is rather scarce. Most literature on optimization of healthcare provision focusses on integrating additional healthcare professions into primary care. However, successful collaborative care approaches make the specialist's expertise and the GP's comprehensive view available to patients (1). Qualitative studies highlight the GPs' role to care for patients with complex care needs. They are an active part of the patients' care continuum in close collaboration with other professions (2). Both, GPs and specialists, are expected to diagnose correctly and are consulted for their disease management skills. GPs tend to focus on general medical and nonmedical problems, whereas the specialists are more familiar with organ specific issues. Therefore, specialists are visited sporadically but usually do not 
continuously manage stable conditions (3). With a GP who controls the patient flow, emergency department visits as well as hospitalizations can be reduced (4). The GP is especially valued for knowledge on the patients' medical history, lifestyle and social environment (5). Continuity of care can be ensured with a GP as a first point of contact for patients in the community and navigating their way through the healthcare system and its institutions (2). Patients "trust their GP to provide competent care, especially when they have more complex healthcare needs" (6).

In comparison to the general population, complex multimorbid patients are more likely to consult other physicians and professionals besides the GP. Since many healthcare professionals (HCPs) are part of these patients' care continuum, oversight is often lost. More often than not there is no commonly shared information system. As a result, there is a lack of comprehensive knowledge on the patients' existing situation $(2,7)$, even though an interoperable health record would be important to improve efficacy and

continuity of care (8). Newly introduced models often focus on one chronic condition (9) but patients with complex chronic disease present a challenging interplay of multiple conditions and symptoms. When providing care to these patients, manifold medical, social and behavioral factors blur primary and secondary care. Typically, such patients require the attention of multiple healthcare providers, facilities or even home-based care (10-12). Therefore, patients with complex chronic conditions are more vulnerable to fragmentation of care and their complex situations can lead to failed care coordination (3). To collaborate successfully, GPs and specialists must overcome local, institutional and healthcare system barriers $(2,13)$.

We conducted a scoping review with the objective to provide an overview of intervention studies that aimed to improve collaboration between GPs and specialists. The review summarizes components and concepts of collaboration, as well as role distributions.

\section{Methods}

We conducted a scoping review $(14,15)$ with the overall goal to provide an overview of improvement strategies. The research question was phrased according to the PICO format (additional file 1) (16). The review follows the recommendations of the PRISMA-ScR checklist (17).

\section{Literature search}

The search strategy consisted of a combination of free text and Medical Subject Headings (MeSH) with Boolean Operators and was structured according to four general concepts: 1) patient population 2) primary care physicians 3) specialized care physicians and 4) type of care provided to the patient. Four relevant articles were used to test whether the search strategy was able to identify suitable literature. Search terms were added until all four articles were identified. Finally, studies were searched in the PubMed database. The final search string is presented in the additional file 2. Last, we identified further relevant literature from the reference lists of selected articles. 


\section{Inclusion criteria}

Original peer-reviewed intervention protocols and studies with a quantitative design published between 2010 and 2020 written in any language were eligible for inclusion in this review. Publications were included if 1) the intervention aimed to improve collaboration between GPs and medical specialists at the primary-secondary interface and 2a) they provided information on components of the interface for shared care and collaboration, or 2 b) defined role distributions between medical specialists and GPs. Articles were excluded if 1 ) the design was not a prospective with either a control group or before-andafter comparison or 2) the intervention involved only other HCPs besides physicians to improve healthcare provision.

\section{Selection}

We extracted potentially eligible literature from the database and checked for inclusion in two steps. First, titles and abstracts were checked if primary and secondary care physicians worked together. Second, full texts were read to ensure that the study sufficiently reported on the collaboration model, as well as the development process and concepts behind it. Two researchers (PL, RT) performed the study selection independently. We discussed studies with a third reviewer (SE) when the selection decision differed. The decision to include was based on consensus among all three researchers.

\section{Data extraction}

Information that contributed to answering the review's research question was extracted into a spreadsheet. The sheet synthesizes key information on each paper (additional file 3). This process was carried out by one researcher (RT). RT and SE regularly discussed the spreadsheet to ensure that it provides on the necessary information.

\section{Results}

As shown in Figure 1, the literature search generated 2,167 potentially eligible publications and further reference screening yielded 7 additional publications. After duplicate removal, 129 full texts were assessed for eligibility. Finally, 30 articles were included in this review. The results are presented as 22 unique projects, which include multiple articles for most projects.

Figure 1: Preferred Reporting Items for Systematic Reviews and Meta-Analyses" (PRISMA) flow diagram of the study selection process (18)

Figure 2 summarizes the review`s results. It visualizes the challenges that influenced the design of interventions, as well as the main components of collaboration and the role distributions identified in the literature. 
Figure 2: Overview of challenges in healthcare provision, components of the interface of collaboration and role distributions as identified from the literature.

HCPs: healthcare professionals; GP: General practitioner.

1: Patient- and disease-related factors refer to challenges in healthcare provision that stem from patient characteristics or characteristics of their conditions. These factors influence the collaboration between specialists and general practitioners, but cannot be modified by the collaboration.

2: Contextual factors originate both from the healthcare system and the collaboration at the primary and secondary care interface, i.e., these factors influence the collaboration between specialists and general practitioners, but can also be modified by this collaboration.

\section{Project characteristics}

\section{Rationale for project}

Most authors designed their intervention with either, challenges in the healthcare system or characteristics of a specific patient population in mind. One important reason was fragmented care, which was a barrier to care provision. Reasons for fragmented care were a lack of communication and cooperation in care that was vulnerable to miss patients at risk. It ultimately resulted in the delayed provision of care $(19,20)$. The lack of unclear roles of involved HCPs, guidelines and referral processes limited the patients' access to care (21-24). Lack of knowledge and awareness of GPs on the condition resulted in a shortcoming of care and preventive measures (25-27). Authors specified that highly prevalent health conditions were focused on in the interventions. Authors reported on the respective condition's burden on healthcare facilities or system resources such as high consultation rates $(23,24$, 28 ) or costs (29-34). Furthermore, projects presented that the health condition of interest is a leading cause of morbidity, hospitalizations and death $(30-33,35-40)$. The current organization of care and its coordination was often considered suboptimal or even outdated for the specific health condition. Finally, the conditions' complexity was mentioned. Interestingly, only few projects reported on what they defined as complex patient care or complex needs even though most of them used this term to describe patientrelated characteristics. These definitions included: polypharmacy $(23,24)$, a specific amount of followups (29), "stage- and symptom-dependent care that comprises multimodal interventions" $(26,27)$, presence of comorbidities $(34,41)$, presence of multiple practitioners (42) and patients' "ongoing management of their [condition] is beyond the capability of their usual $G P^{\prime \prime}(43,44)$.

\section{Origin and study design}

Of the 22 original projects, five were located in the United States $(19,23,24,31-33,35-37,45,46)$, four in Canada $(21,22,29,30,42)$; two projects, respectively, were located in Australia $(43,44,47)$, Brazil (41), 
Spain $(28,48)$, Switzerland $(26,27,38)$ and the Netherlands $(20,49)$. One project was based in Germany (25), Italy (34) and New Zealand (39).

Eight projects applied a before-and-after design with outcome measurements pre and post intervention $(19,23,24,30-34,41,45)$, whereof three before-and-after designs further compared the intervention group to a control group $(38,47,48)$. Additionally, three projects compared the intervention group to a control group, which was usual care $(28,42-44)$. Five projects were RCTs $(20,26,27,35-37,39,49)$. One project used an array of methods for their evaluation; the authors applied a randomized stepped-wedge design, a before-and-after and a controlled design separately $(21,22)$. Furthermore, two protocols for RCTs $(29,46)$ and one protocol for a before-and-after design (25) were included in this review.

Seven projects applied randomization, and of those, two projects randomize on a patient level $(21,22,35$ $37)$ and five on a healthcare provider level (e.g. long-term care home, GP practice) $(20,26,27,29,39,49)$. Three projects blinded the involved healthcare professionals (HCPs) with regard to the allocation sequence $(21,22,35-37,39)$ one mentioned specifically that blinding was not possible $(21,22)$ and three gave no information whether any study participants were blinded $(20,26,27,46,49)$.

Twelve projects reported an intervention period of 12 months $(20-22,26,27,29,30,38,42-47,49)$ and one project of 10 months $(23,24)$. Nine projects evaluated the intervention for more than one year with a mean duration of approximately 40 months, ranging from 15 to 156 months $(19,25,28,31-34,39,41$, 48).

\section{Patient population}

Projects involved various patient populations with either specific or unspecific diagnoses. Specific diagnoses included kidney diseases in one (42) and specifically at a chronic stage (chronic kidney disease (CKD)) in five projects $(19,20,30-34)$. Furthermore, three projects included patients diagnosed with chronic obstructive pulmonary disorder (COPD) $(26,27,38,45)$, whereas a combination of asthma and COPD (41), chronic hepatitis B (CHB) (47), chronic heart failure (CHF) (35-37) and coronary artery disease (CAD) (46) were investigated in one project. A diagnosis of diabetes type 2 was focused on in two projects $(43,44,49)$. One project defined the patient population of interest as a mix of several specific diagnoses, where at least one of the following was present: diabetes, cardiovascular disease, COPD, asthma or cardiovascular risk factors $(21,22)$. Six projects characterized their patient population independent of diagnoses such as long-term $(29,39)$, end of life care (25) or care for patients with multiple chronic conditions $(23,24)$. Furthermore, one project focused on heart diseases in general (48) and another on gastroenterology and hepatology without specific diagnoses (28).

\section{Organization of care and components of interface}


Interventions implemented process and system changes to enhance collaboration between primary and secondary care. The measures focused on education and knowledge transfer, as well as shifting responsibilities to additional HCPs.

\section{Education and knowledge transfer}

Education and training for GPs and primary care staff was widely applied. Two of the included projects solely designed continuous medical education (CME) programs as the intervention. Adams et al. created a CME program that targeted primary care providers and aimed to improve their knowledge/comprehension, self-confidence, and, ultimately, clinical practice (45). Uniquely, Pang et al. developed a mentor-mentee program with individual training sessions, regular regional learning meetings and case consultations. The educational aspect of the intervention focused on early detection and identification of patients with CKD (30). A Dutch project focused on educating primary care staff in "blood pressure management and treatment, cholesterol lowering, blood-glucose management, and lifestyle advice" for patients with $\operatorname{CKD}(19,23,24,28,29,49)$. Fortin et al. arranged a chronic disease prevention program with a focus on patients' self-management. Included HCPs received training in motivational interviewing to help reduce the burden of chronic diseases and related high-risk lifestyles $(21,22)$.

Most projects combined educational programs with other components in the intervention. Tinetti et al. encouraged physicians to review selected patient cases together and to identify patient priorities besides offering CME. The physicians were stimulated to align their care towards patient priorities and the same outcomes $(23,24)$. Health condition specific education was part of Martins' et al. study to improve the physicians' knowledge before inviting a medical specialist to perform joint consultations in the GP practice $(34,41)$. Before opening specialized outpatient clinics, Santoro et al. provided teaching. Especially, early identification of the at-risk population, effective prevention and treatment strategies and inappropriate drug utilization were taught to GPs. Within the outpatient facilities GPs were then asked to profile patients, assess their co-morbidities and renal insufficiency stage, plan lab tests and referrals. Furthermore, if appropriate, GPs developed personalized therapy and nutrition plans $(34,41)$.

Care arrangements between GPs and specialists were usually based on knowledge transfer and hands-on teaching without planning formal education events $(19,23,24,28,29,49)$. As an example, Falces et al., integrated a cardiologist into a primary care practice to support follow-up (48). Similarly, the project of Hynes and Porter introduced new HCPs in the patient-centered medical home, who shared their information in multidisciplinary team meetings $(31-33,38)$. In an Italian study, co-management of patients was encouraged and the specialist was involved more, as soon as the GP needed assistance in the management (34). Several interventions provided screening tools (19), guidelines (19, 25-27), treatment protocols (47), standardized discharge processes (46) and defined referral procedures $(19,26$ $28,42,47,48)$. Usually, these standards were developed before the project and in accordance with project participants and experts to ensure applicability. 
Electronic consultation tools were mainly introduced to provide remote assistance for decision-making and support by specialists for GPs. Ong et al. established a co-management arrangement which was virtual and supported with guiding referral processes that visualized patients' pathways (42). Other electronic platforms were incorporated to store patients' health records (20), exchange documents (42), and facilitate direct interaction $(29,46)$. Ho et al. focused on providing telemedicine consultation for GPs and provided educational documents on evidence-based care of geriatric patients $(29,46)$. Reasons for integrating e-consultation tools included improved specialist access and reductions in unnecessary inperson referrals $(29,42)$. Furthermore, they added diagnostic and therapeutic support as well as a way to provide continuing health education (46).

\section{Shifting responsibilities to other HCPs}

Additional HCPs besides physicians were widely integrated at the primary-secondary interface. Bekelman et al., Connolly et al. and Porter et al. integrated nurse coordinators or specialized nurses to arrange multidisciplinary team meetings (39). Additionally, Porter et al. added a community health worker and a pharmacist to the team in a long-term care home (31-33) and Bekelman et al. included a social worker to perform psychosocial assessments (35-37).

Their roles varied between projects concerning their involvement in the patients' care processes. Nurse practitioners, nurse educators, specialized nurses, nurse coordinators and practice nurses were the professionals most included in interventions. Their level of involvement varied, but nurses engaged rather actively and performed patient assessments, developed and reviewed care plans, coached staff, took over patient education and shared responsibilities with GPs $(19,21,22,30-33,35-39,43,44,47,49)$. Other HCPs with active roles included pharmacists, physiotherapists and community health workers who provided patient education on profession-specific care $(31-33,38)$. In a Canadian project, the research staff was engaged actively and performed pharmacological consultations and medication reviews (29). Rather passive involvement in the care provision included medical education of nurses, physician assistants, medical students, respiratory physiotherapists without any further specified role $(26-28,45$, 46).

\section{Role distributions}

Some projects defined roles prior to the intervention and others openly gave physicians the task to define them themselves. The following synthesizes the roles, how they were distributed among GPs and specialists or shared between them.

Many interventions assigned clearly distinct roles to GPs and specialists. The more severe the disease and symptoms, the more likely a specialist was involved in patient care. On the other hand, the GP was often expected to support with problems of little to moderate severity. Accordingly, the patients were referred to the specialist for specialized assessments and treatments $(23-27,34,47)$. Furthermore, several 
projects expected specialists to provide medical education to GPs. GPs were then expected to enhance their knowledge and services by receiving $\operatorname{CME}(23,24,26,27,30,43,44)$. Pang et al. assigned GPs to specialists, acting as mentors. In brief, an assigned mentor was responsible for regular education sessions and offered one-on-one consultations, but little supervision during daily practice. In this project, the GPs knowledge on diagnosing and managing patients with CKD was evaluated (30). Another project encouraged a hospital to distribute specialized patient care plans to the GPs upon referral. Management was transferred to the GPs but overseen with an electronic treatment protocol by the hospital team. The primary care team was evaluated regarding their ability to treat and monitor patients with CKD (47). In a Swiss project, the GP was asked to follow a checklist closely and their adherence was monitored by the research team $(26,27)$.

Other studies included interventions on joint consultations or patient case discussions. They allowed for more direct interaction between physicians $(20,29,41,42,46)$. For instance, van Gelder et al. integrated a web-based consultation tool to enable GPs to align their care with nephrologists by consulting them. The GP was responsible for patient care and data entry into patients' electronic medical records. Specialists had access to the same information and provided streamlined advice (20). Falces et al. integrated a specialist into the primary care team as a new member to assist in patients' follow-up and to distribute the patients between care levels. The physicians were able to establish their own referral criteria and actively discuss their different responsibilities. In this case, specialists provided medical advice when needed, and had weekly consultation sessions with GPs (48). Even though the specialist took the lead in joint consultations with the GP and patients in a Spanish study, the GP was still the main person of contact for the patient (28). In the project of Fortin et al., GPs were in charge of long-term follow-up of patients, while the specialist took a rather passive role and had regular contact with the GP for support accordingly $(21,22)$. Haley et al. motivated GPs and specialists to develop tools addressing specific barriers and root causes for problems related to collaboration. In this project, the GP and specialist were considered equally important for patient care and co-managed within the scope of their expertise. The GP was in charge of patient management and sought specialized support when needed (19).

In projects that integrated further HCPs into primary care institutions, the GP was expected to supervise and to provide support. The GP was usually in charge and responsible for patient care but delegated certain tasks and responsibilities (31-33, 35-38, 43, 44, 49). Compared to a traditional primary care setting, a project taking place in a long-term care home is co-managed by the GP and other HCPs without the GP taking the lead or being superordinate (39).

\section{Outcomes and results of projects}

The majority of projects analyzed patient-related outcomes. Overall, the results were positive and reported improvements in outcomes. Several projects reported on improvements in patients' health conditionspecific outcomes. They included $\mathrm{HbA1c}$ level $(43,44)$, blood pressure (49) or estimated glomerular filtration rate $(20,34)$. Falces et al. declared that an improvement in body mass index, better control of 
blood pressure and cholesterol were achieved (48). No change was reported in the primary outcome, a patient-reported disease-specific HRqoL questionnaire, but secondary outcomes (depression, fatigue and anxiety) improved (35-37). Two projects evaluated referral rates to the specialist and found a decrease at project termination $(28,41)$. Connolly's et al. measured a decrease in avoidable emergency department admissions (39).

Self-reported physician outcomes included improved clinician confidence (45), satisfaction (48), increased knowledge $(30,45)$, heightened attention to communication and awareness for a health condition (19). Furthermore, the participation rate of GPs in attending one-on-one case discussions with specialists was reported (30). Additionally, process-related outcomes included improved documentation of outcomes by the GP (19) and an improved disease-specific medication dispensing (41). Finally, some interventions were reported to increase costs slightly (20) or to not increase resources (48).

\section{Discussion}

\section{Main findings}

This review provides a broad insight into interventions aiming to improve collaboration between GPs and medical specialists at the primary and secondary care interface. There was no gold standard on the measures and different process and system related changes were implemented. Most interventions showed that GPs are able to take on additional responsibilities successfully with streamlined specialist support. The importance of GPs as a major point of contact for patients was emphasized in various healthcare conditions and settings.

\section{Implications for development of care models}

All projects were able to identify challenges in collaboration that were addressed in the interventions accordingly. This finding might seem obvious, but stakeholders should be aware that interventions could be "overdesigned". A thorough analysis of the situation should be performed to prevent interventions from losing focus trying to address too many issues. In fact, identified projects rarely created new care models and rather extended existing ones with additional measures. Measures on process and system levels were used and seemed to achieve positive results. In particular, process-related changes such as shared education and checklists, to better manage and coordinate, seemed to be implemented easily and showed changes for clinicians and researchers quickly. Joint patient consultations, adding new HCPs with specific responsibilities or the implementation of web-based tools or telemedicine as examples for system changes, needed a larger commitment from involved parties. Nonetheless, implementing a central triage between institutions was more likely to re-organize the model of care than other interventions. Of course, the generalizability of these interventions is not given. Presented measures cannot simply be integrated into daily practice to achieve the same results, as contextual factors most likely differ. 
Therefore, it should be stressed again that an analysis of the collaboration prior to designing an intervention is essential.

More than half of the projects had a duration of below or equal to twelve months, which might be rather short to analyze quality of healthcare provision or knowledge implementation. Longitudinal study designs make sense, as these types of interventions are related to transitional processes (11). The interventions should be replicated in other settings and tested in clinical practice to confirm the findings. As mentioned before, only few projects gave a definition of complexity. Researchers should be aware that this concept is not used consistently among the scientific community and providing an understanding on how the term was defined is essential for shaping care models.

\section{Limitations and strengths}

The review has some limitations. It is likely that relevant published articles were not identified as the review includes literature from only one database published between 2010 and 2020. We also realize that there might be unpublished projects from medical societies or governments, but the amount of published literature seems to be sufficient to provide an overview of the approaches. Furthermore, the comparability of included study protocols may be limited regarding projects that already reported results. These protocols were included, as we wanted to focus on concepts in a broader health system perspective in this review instead of summarizing outcomes or effect sizes of interventions. Moreover, the settings and outcomes of projects vary widely and the mix is sometimes hard to interpret and compare. In particular, projects with GP-specialist collaboration as one part of a larger intervention, limit the possibility to determine the model parameters of interest. For example, many of the interventions assign responsibilities to other HCPs besides physicians, which presumably influences collaboration at the primary-secondary care interface, as well. Lastly, the reporting of roles and responsibilities effects our result synthesis. Authors should consider that their reporting informs other researchers applying similar concepts, who highly benefit from a detailed description of processes designing the intervention. Besides the listed limitations, this scoping review provides an informative overview for researchers, HCPs, policy makers and other stakeholders wishing to improve collaboration between specialists and GPs. The presented measures were shown to achieve this goal.

\section{Conclusion}

Interventions to improve collaboration between GPs and medical specialists seem promising. Further efforts should be made to test and apply the findings in broad clinical practice.

\section{Abbreviations}

CME: continuous medical education

GP: general practitioner 
HCP: healthcare professional

MeSH: medical subject headings

RCT: randomized controlled trial

\section{Declarations}

\section{Ethics approval and consent to participate}

Not applicable.

\section{Consent for publication}

Not applicable.

\section{Availability of data and materials}

All data generated or analyzed during this study are included in this published article and its supplementary information files.

\section{Competing interests}

The authors declare that they have no competing interests.

\section{Funding}

The authors receive no specific funding for the study.

\section{Authors' contributions}

RT and SE designed the research question, search strategy and wrote the main parts of the manuscript. $\mathrm{PL}$ and RT performed the study selection and RT analyzed the data. AS, AG and CM reviewed the manuscript and gave feedback concerning their field of expertise which are specialized medicine, health services research and primary care accordingly. All authors read and approved the final manuscript.

\section{Acknowledgements}

The authors would like to thank Allina Lopez for correcting the manuscript. 


\section{References}

1. Janssen M, Sagasser MH, Fluit C, Assendelft WJJ, de Graaf J, Scherpbier ND. Competencies to promote collaboration between primary and secondary care doctors: an integrative review. BMC family practice. 2020;21(1):179.

2. Loeb DF, Bayliss EA, Candrian C, deGruy FV, Binswanger IA. Primary care providers' experiences caring for complex patients in primary care: a qualitative study. BMC family practice. 2016;17:34.

3. Piterman L, Koritsas S. Part I. General practitioner-specialist relationship. Internal medicine journal. 2005;35(7):430-4.

4. Siu HY, Steward N, Peter J, Cooke L, Arnold DM, Price D. A novel primary-specialist care collaborative demonstration project to improve the access and health care of medically complex patients. Chronic Illn. 2017;13(3):151-70.

5. Cheraghi-Sohi S, Bower P, Mead N, McDonald R, Whalley D, Roland M. What are the key attributes of primary care for patients? Building a conceptual 'map' of patient preferences. Health expectations : an international journal of public participation in health care and health policy. 2006;9(3):275-84.

6. Anvik T, Holtedahl KA, Mikalsen H. "When patients have cancer, they stop seeing me"-the role of the general practitioner in early follow-up of patients with cancer-a qualitative study. BMC family practice. 2006;7:19.

7. Berendsen AJ, Kuiken A, Benneker WH, Meyboom-de Jong B, Voorn TB, Schuling J. How do general practitioners and specialists value their mutual communication? A survey. BMC health services research. 2009;9:143.

8. Rosemann T, Wensing M, Rueter G, Szecsenyi J. Referrals from general practice to consultants in Germany: if the GP is the initiator, patients' experiences are more positive. BMC health services research. 2006;6:5.

9. Doessing A, Burau V. Care coordination of multimorbidity: a scoping study. J Comorb. 2015;5:15-28.

10. Sevick MA, Trauth JM, Ling BS, Anderson RT, Piatt GA, Kilbourne AM, et al. Patients with Complex Chronic Diseases: perspectives on supporting self-management. Journal of general internal medicine. 2007;22 Suppl 3:438-44.

11. van Hoof SJM, Quanjel TCC, Kroese M, Spreeuwenberg MD, Ruwaard D. Substitution of outpatient hospital care with specialist care in the primary care setting: A systematic review on quality of care, health and costs. PloS one. 2019;14(8):e0219957.

12. Loeb DF, Binswanger IA, Candrian C, Bayliss EA. Primary care physician insights into a typology of the complex patient in primary care. Annals of family medicine. 2015;13(5):451-5.

13. Foster M, Burridge L, Donald M, Zhang J, Jackson C. The work of local healthcare innovation: a qualitative study of GP-led integrated diabetes care in primary health care. BMC health services research. 2016;16:11.

14. Munn Z, Peters MDJ, Stern C, Tufanaru C, McArthur A, Aromataris E. Systematic review or scoping review? Guidance for authors when choosing between a systematic or scoping review approach. 
BMC medical research methodology. 2018;18(1):143.

15. Lockwood C, Dos Santos KB, Pap R. Practical Guidance for Knowledge Synthesis: Scoping Review Methods. Asian Nurs Res (Korean Soc Nurs Sci). 2019;13(5):287-94.

16. Eriksen MB, Frandsen TF. The impact of patient, intervention, comparison, outcome (PICO) as a search strategy tool on literature search quality: a systematic review. J Med Libr Assoc. 2018;106(4):420-31.

17. Tricco AC, Lillie E, Zarin W, O'Brien KK, Colquhoun H, Levac D, et al. PRISMA Extension for Scoping Reviews (PRISMA-ScR): Checklist and Explanation. Annals of internal medicine. 2018;169(7):467-73.

18. Moher D, Liberati A, Tetzlaff J, Altman DG, Group P. Preferred reporting items for systematic reviews and meta-analyses: the PRISMA statement. PLoS medicine. 2009;6(7):e1000097.

19. Haley WE, Beckrich AL, Sayre J, McNeil R, Fumo P, Rao VM, et al. Improving care coordination between nephrology and primary care: a quality improvement initiative using the renal physicians association toolkit. American journal of kidney diseases : the official journal of the National Kidney Foundation. 2015;65(1):67-79.

20. van Gelder VA, Scherpbier-de Haan ND, van Berkel S, Akkermans RP, de Grauw IS, Adang EM, et al. Web-based consultation between general practitioners and nephrologists: a cluster randomized controlled trial. Family practice. 2017;34(4):430-6.

21. Fortin M, Chouinard MC, Bouhali T, Dubois MF, Gagnon C, Belanger M. Evaluating the integration of chronic disease prevention and management services into primary health care. BMC health services research. 2013;13:132.

22. Fortin M, Chouinard MC, Dubois MF, Belanger M, Almirall J, Bouhali T, et al. Integration of chronic disease prevention and management services into primary care: a pragmatic randomized controlled trial (PR1MaC). CMAJ Open. 2016;4(4):E588-E98.

23. Blaum CS, Rosen J, Naik AD, Smith CD, Dindo L, Vo L, et al. Feasibility of Implementing Patient Priorities Care for Older Adults with Multiple Chronic Conditions. Journal of the American Geriatrics Society. 2018;66(10):2009-16.

24. Tinetti ME, Esterson J, Ferris R, Posner P, Blaum CS. Patient Priority-Directed Decision Making and Care for Older Adults with Multiple Chronic Conditions. Clin Geriatr Med. 2016;32(2):261-75.

25. Afshar K, Muller-Mundt G, van Baal K, Schrader S, Wiese B, Bleidorn J, et al. Optimal care at the end of life (OPAL): study protocol of a prospective interventional mixed-methods study with pretestposttest-design in a primary health care setting considering the view of general practitioners, relatives of deceased patients and health care stakeholders. BMC health services research. 2019;19(1):486.

26. Markun S, Rosemann T, Dalla-Lana K, Steurer-Stey C. Care in Chronic Obstructive Lung Disease (CAROL): a randomised trial in general practice. The European respiratory journal. 2018;51(5).

27. Steurer-Stey C, Markun S, Lana KD, Frei A, Held U, Wensing M, et al. The improving care in chronic obstructive lung disease study: CAROL improving processes of care and quality of life of COPD patients in primary care: study protocol for a randomized controlled trial. Trials. 2014;15:96. 
28. Mata-Roman L, del Olmo-Martinez L, Briso-Montiano R, Garcia-Pascual A, Caton-Valdes M, JimenezRodriguez-Vila $\mathrm{M}$, et al. Periodic gastroenterology and hepatology meetings with primary care. Reasons for consultation. Revista espanola de enfermedades digestivas : organo oficial de la Sociedad Espanola de Patologia Digestiva. 2013;105(9):521-8.

29. Ho JM, Tung J, Maitland J, Mangin D, Thabane L, Pavlin JM, et al. GeriMedRisk, a telemedicine geriatric pharmacology consultation service to address adverse drug events in long-term care: a stepped-wedge cluster randomized feasibility trial protocol (ISRCTN17219647). Pilot Feasibility Stud. 2018;4:116.

30. Pang J, Grill A, Bhatt M, Woodward GL, Brimble S. Evaluation of a mentorship program to support chronic kidney disease care. Canadian family physician Medecin de famille canadien. 2016;62(8):e441-7.

31. Hynes DM, Fischer M, Fitzgibbon M, Porter AC, Berbaum M, Schiffer L, et al. Integrating a Medical Home in an Outpatient Dialysis Setting: Effects on Health-Related Quality of Life. Journal of general internal medicine. 2019;34(10):2130-40.

32. Hynes DM, Fischer MJ, Schiffer LA, Gallardo R, Chukwudozie IB, Porter A, et al. Evaluating a novel health system intervention for chronic kidney disease care using the RE-AIM framework: Insights after two years. Contemporary clinical trials. 2017;52:20-6.

33. Porter AC, Fitzgibbon ML, Fischer MJ, Gallardo R, Berbaum ML, Lash JP, et al. Rationale and design of a patient-centered medical home intervention for patients with end-stage renal disease on hemodialysis. Contemporary clinical trials. 2015;42:1-8.

34. Santoro A, Gibertoni D, Rucci P, Mancini E, Bonucchi D, Buscaroli A, et al. The PIRP project (Prevenzione Insufficienza Renale Progressiva): how to integrate hospital and community maintenance treatment for chronic kidney disease. Journal of nephrology. 2019;32(3):417-27.

35. Bekelman DB, Allen LA, McBryde CF, Hattler B, Fairclough DL, Havranek EP, et al. Effect of a Collaborative Care Intervention vs Usual Care on Health Status of Patients With Chronic Heart Failure: The CASA Randomized Clinical Trial. JAMA internal medicine. 2018;178(4):511-9.

36. Bekelman DB, Hooker S, Nowels CT, Main DS, Meek P, McBryde C, et al. Feasibility and acceptability of a collaborative care intervention to improve symptoms and quality of life in chronic heart failure: mixed methods pilot trial. Journal of palliative medicine. 2014;17(2):145-51.

37. Bekelman DB, Plomondon ME, Sullivan MD, Nelson K, Hattler B, McBryde C, et al. Patient-centered disease management (PCDM) for heart failure: study protocol for a randomised controlled trial. BMC cardiovascular disorders. 2013;13:49.

38. Carron T, Bridevaux PO, Lorvall K, Parmentier R, Moix JB, Beytrison V, et al. Feasibility, acceptability and effectiveness of integrated care for COPD patients: a mixed methods evaluation of a pilot community-based programme. Swiss medical weekly. 2017;147:w14567.

39. Connolly MJ, Broad JB, Bish T, Zhang X, Bramley D, Kerse N, et al. Reducing emergency presentations from long-term care: A before-and-after study of a multidisciplinary team intervention. Maturitas. 2018;117:45-50. 
40. Bartlett M, Wang J, Hay L, Pang G. Health service use in the older person with complex health needs. Australian health review : a publication of the Australian Hospital Association. 2019;43(1):62-70.

41. Martins SM, Salibe-Filho W, Tonioli LP, Pfingesten LE, Braz PD, McDonnell J, et al. Implementation of 'matrix support' (collaborative care) to reduce asthma and COPD referrals and improve primary care management in Brazil: a pilot observational study. NPJ primary care respiratory medicine. 2016;26:16047.

42. Ong SW, Kaushal A, Pariser P, Chan CT. An Integrated Kidney Care eConsult Practice Model: Results from the iKinect Project. American journal of nephrology. 2019;50(4):262-71.

43. Askew DA, Jackson CL, Ware RS, Russell A. Protocol and baseline data from The Inala Chronic Disease Management Service evaluation study: a health services intervention study for diabetes care. BMC health services research. 2010;10:134.

44. Russell AW, Baxter KA, Askew DA, Tsai J, Ware RS, Jackson CL. Model of care for the management of complex Type 2 diabetes managed in the community by primary care physicians with specialist support: an open controlled trial. Diabet Med. 2013;30(9):1112-21.

45. Adams SG, Pitts J, Wynne J, Yawn BP, Diamond EJ, Lee S, et al. Effect of a primary care continuing education program on clinical practice of chronic obstructive pulmonary disease: translating theory into practice. Mayo Clinic proceedings. 2012;87(9):862-70.

46. Batista J, Furtado MV, Katz N, Agostinho MR, Neto BS, Harzheim E, et al. Telemedicine-supported transition of stable coronary artery disease patients from tertiary to primary health care facilities: protocol for a randomized non-inferiority trial. BMC health services research. 2016;16:227.

47. O'Leary DA, Cropp E, Isaac D, Desmond PV, Bell S, Nguyen T, et al. "B in IT" - a community-based model for the management of hepatitis B patients in primary care clinics using a novel web-based clinical tool. Hepatology, medicine and policy. 2018;3:1.

48. Falces C, Andrea R, Heras M, Vehi C, Sorribes M, Sanchis L, et al. [Integration between cardiology and primary care: impact on clinical practice]. Revista espanola de cardiologia. 2011;64(7):564-71.

49. Scherpbier-de Haan ND, Vervoort GM, van Weel C, Braspenning JC, Mulder J, Wetzels JF, et al. Effect of shared care on blood pressure in patients with chronic kidney disease: a cluster randomised controlled trial. Br J Gen Pract. 2013;63(617):e798-806.

\section{Figures}



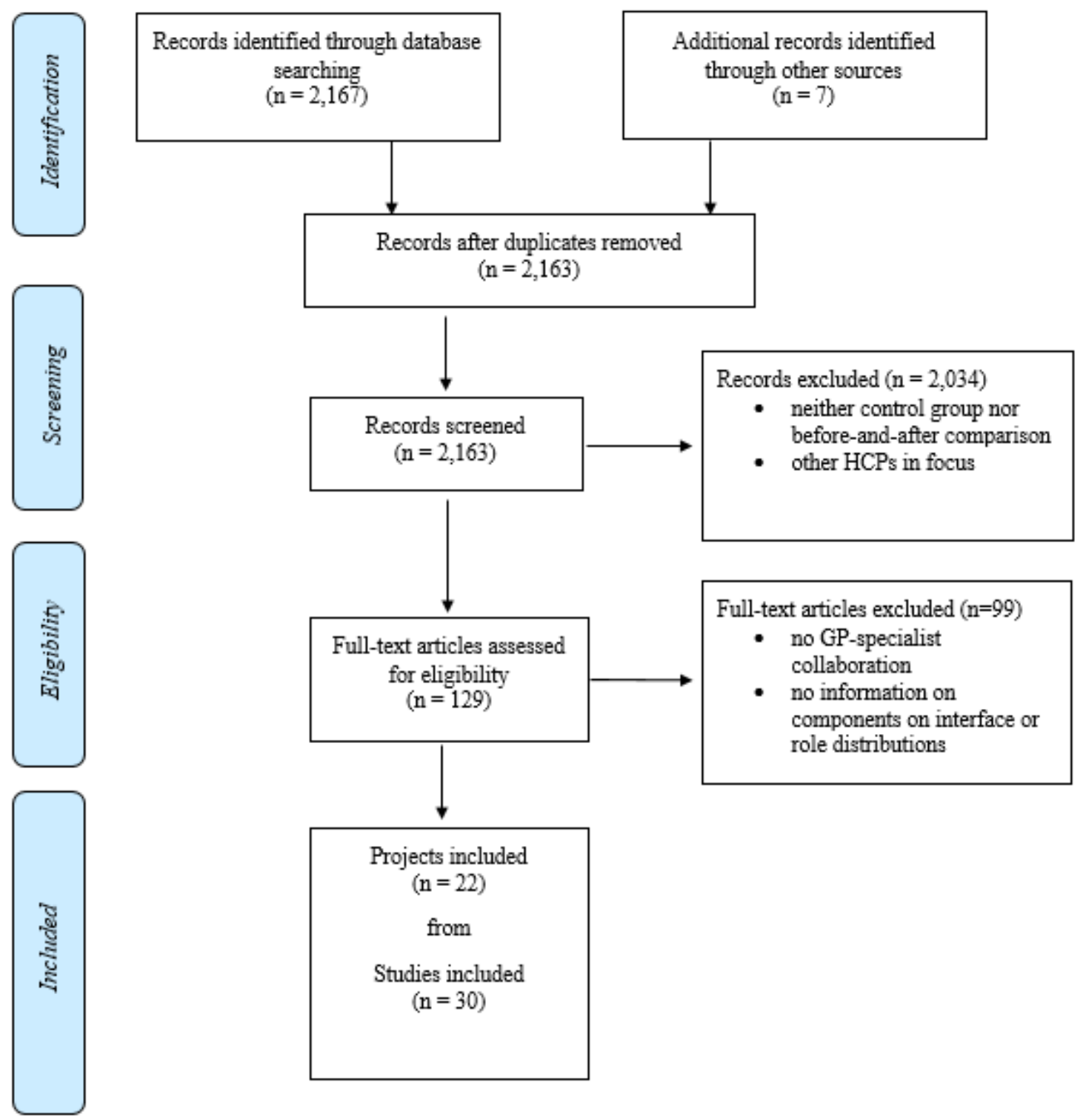

Projects included

$(\mathrm{n}=22)$

from

Studies included

$(\mathrm{n}=30)$

\section{Figure 1}

Preferred Reporting Items for Systematic Reviews and Meta-Analyses" (PRISMA) flow diagram of the study selection process (18) 
Patient- and disease-related factors ${ }^{1}$

Chronic condition

Multimorbidity

High prevalence

Medical specialists
Specialist roles

Provide specialized support for assessments and treatment

Inform GP post-consultation

Suggest treatment to GP
Components of the interface

Education and knowledge transfer

Provide medical education

Meet team regularly

Re-locate specialist into primary care

Apply joint consultations

Use telemedicine

Shifting responsibilities to other HCPS

Add HCPs to the care team

Defining role distributions

Agree on patient pathways

Define referral processes

Establish designated outpatient facilities

Identified roles

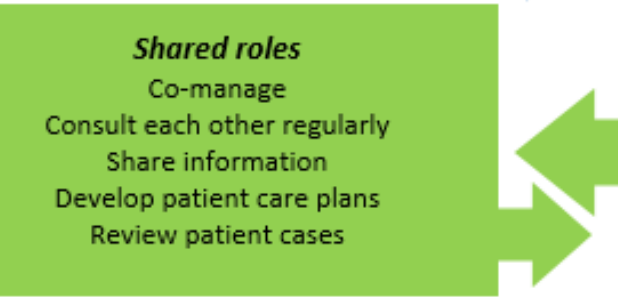

Contextual factors ${ }^{2}$

Fragmentation of care

Missing coordination of care

Unclear roles

Lack of communication

Restricted access
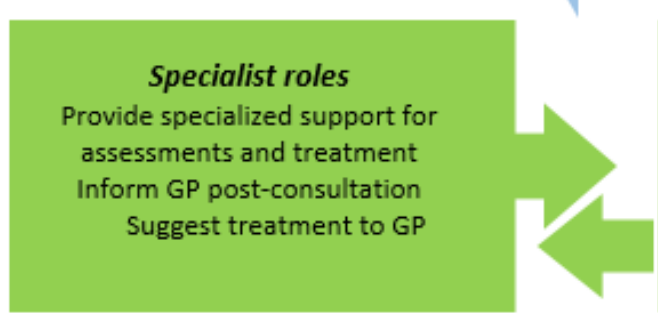

GP roles

Select patients for referral to specialist Supervise patient management Provide long-term follow-up Supervise and support other HCPs Co-manage with other HCPs

\section{Figure 2}

Overview of challenges in healthcare provision, components of the interface of collaboration and role distributions as identified from the literature.

\section{Supplementary Files}

This is a list of supplementary files associated with this preprint. Click to download.

- Additionalfile1.pdf

- Additionalfile2.pdf

- Additionalfile3.pdf 